\title{
Optimal Stochastic Sensor Error Modeling based on Actual Impact on Quality of GNSS-INS Integrated Navigation
}

\author{
Mehran Khaghani, University of Geneva (UNIGE) \\ Stéphane Guerrier, University of Geneva (UNIGE) \\ Jan Skaloud, Swiss Federal Institute of Technology in Lausanne (EPFL) \\ Yuming Zhang, University of Geneva (UNIGE)
}

\begin{abstract}
Proper modeling of stochastic errors in inertial sensors plays a crucial role in the achievable quality of GNSS-INS integration especially with low-cost inertial sensors. Generalized Method of Wavelet Moments (GMWM) can model the underlying process for such errors with arbitrarily complex structure to obtain close match to the observed errors in terms of wavelet variance. In comparison to the widely used and IEEE adopted error modeling using Allan Variance, this method provides consistent estimation, ability to estimate parameters of composite stochastic models of much higher complexities, and considerably easier usage. However, the level of improvement in navigation quality does not necessarily grow proportionally to the fidelity of the error models. Therefore, opting for unnecessarily complex models may only increase computational load with no tangible gain in navigation quality. On the other hand, converging estimation of higher complexity models, generally speaking, requires longer estimation periods and higher dynamics to improve observability of error states. This implies yet another motivation to find an optimal sensor error model avoiding unnecessary complexities. In this paper, we employ two methods to investigate the effect of model complexity on integrated navigation performance. Firstly, a covariance propagation is performed on static conditions, as is the standard scenario in error analysis of inertial navigation systems. Afterwards, an emulation study is performed based on a real Unmanned Aerial Vehicle (UAV) flight and error signals of a Navchip V2 Inertial Measurement Unit (IMU). Results of both methods are in general agreement, and suggest that more complex models in general provide higher accuracy for the navigation system and a more consistent covariance prediction by the navigation filter. This difference is, however, more noticable only in GNSS outages of longer duration (tens of seconds). However, the benefits of more complex models may be only marginal in other applications, depending on the duration of inertial coasting and availability of other sensory data such as GNSS observations.
\end{abstract}

\section{INTRODUCTION}

In design and implementation of integrated INS-based navigation systems, proper knowledge and modeling of inertial sensor errors is an important key to provide performant and reliable navigation systems [1]. Inertial sensors, especially those of lower grades, can have very complex error models, identification of which requires proper and modern tools. Generalized method of wavelet moments (GMWM) is a rather recent method [2]-[4] for identification of a probabilistic time series model and was especially optimized for modeling stochastic errors in inertial sensors. There is an open source R package to use GMWM [5], as well as a publicly available free web tool to facilitate its usage even further [6]. Compared to Allan variance slope method, which is the norm in industry and adopted by IEEE as a standard [7] for this purpose, GMWM is consistent estimator and is very flexible in identifying highly complex models such as the ones including several first order Gauss-Markov processes, not to forget its ease of usage due to high degree of automation. Such flexibility may be tempting toward adopting unnecessarily complex models neglecting the application requirements and potential benefits of higher fidelity models.

This paper aims to present a short study on the effect of employing IMU error models of different complexities in integrated INSbased navigation. As a first and more general approach, a covariance matrix analysis is performed in static conditions, which is a standard scenario for error analysis of inertial navigation systems [1], [8]. In addition, an emulation work flow is introduced and employed to extend the study to more specific real-world applications, where a real trajectory and real sensor errors are used to study the effect of various IMU error models of different complexity on navigation solution accuracy and consistency of predicted covariances by the navigation filter. 


\section{IMU AND ITS REFERENCE ERROR MODEL}

To demonstrate the proposed analyzes we have employed data from small MEMS IMU called Navchip V2 [9] that represent a high quality in its category. Static signals were collected during 6 hours at $200 \mathrm{~Hz}$ and their variation was analyzed using GMWM. We first fitted a model of high fidelity that serves hereinafter as the modeling reference representing correctly the stochastic behavior of underlying error signals. It is worth mentioning that the remaining minor imperfections of this model have no effect on the subsequent analysis, as the performance of all the models is compared to this reference model regardless the quality of this representation (remaining albeit high). Similar analysis can be applied to different IMUs, and the specific model employed here is used only as a demonstrator. Figure 1 shows the wavelet variance plot for the observed noise and the reference model fitted by GMWM. The model parameters are depicted in Table 1 . The reference model comprises of a white noise and 3 correlated processes $\left(1^{\text {st }}\right.$ order GaussMarkov) for both accelerometers and gyroscopes, plus a drift for the accelerometers.
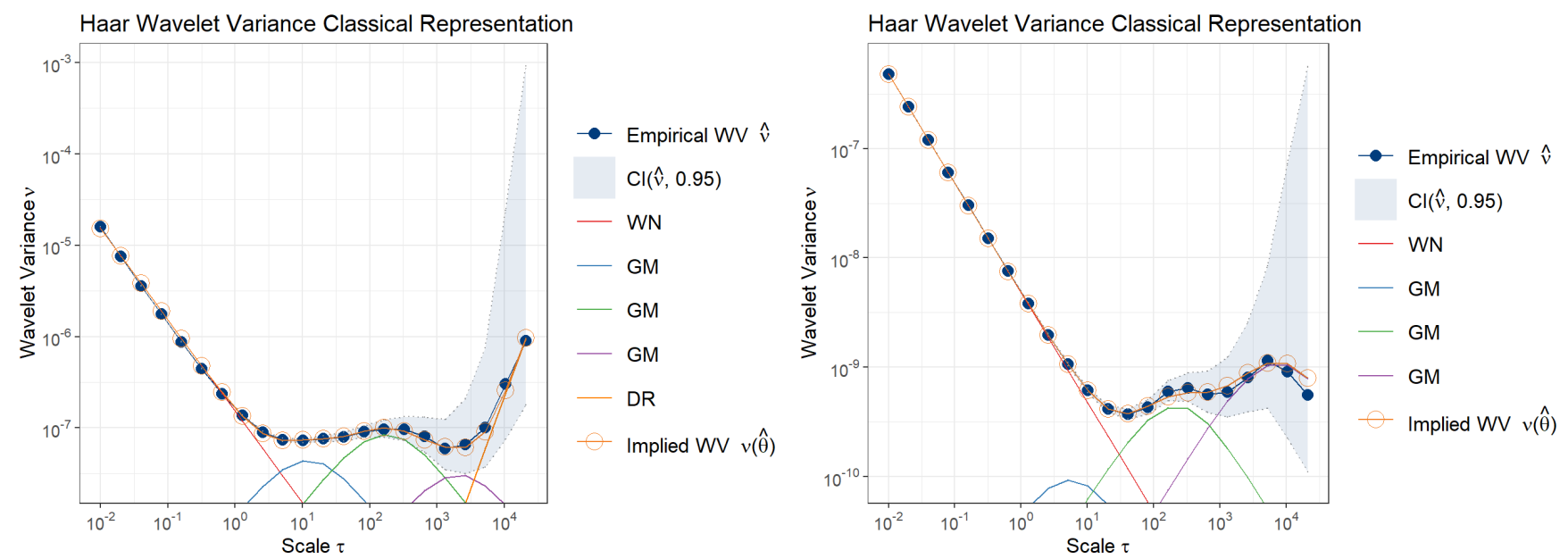

Figure 1: Wavelet Variance plots of the real IMU data (blue line w. full circles) and the reference model (orange line w. empty circles) for accelerometers (left) and gyroscopes (right)

\section{ERROR MODEL CANDIDATES}

In addition to the reference model for the IMU at hand we consider three other "sub-optimal" models with different levels of complexity and therefore "fitting fidelity". The GMWM plots of these models are shown in Figure 2 to Figure 4 and their parameters are presented in Table 1. As shown in this table Model 1 comprises of white-noise only, while Model 2 and Model 3 of different combination of white-noise and correlated processes including a drift for accelerometers.
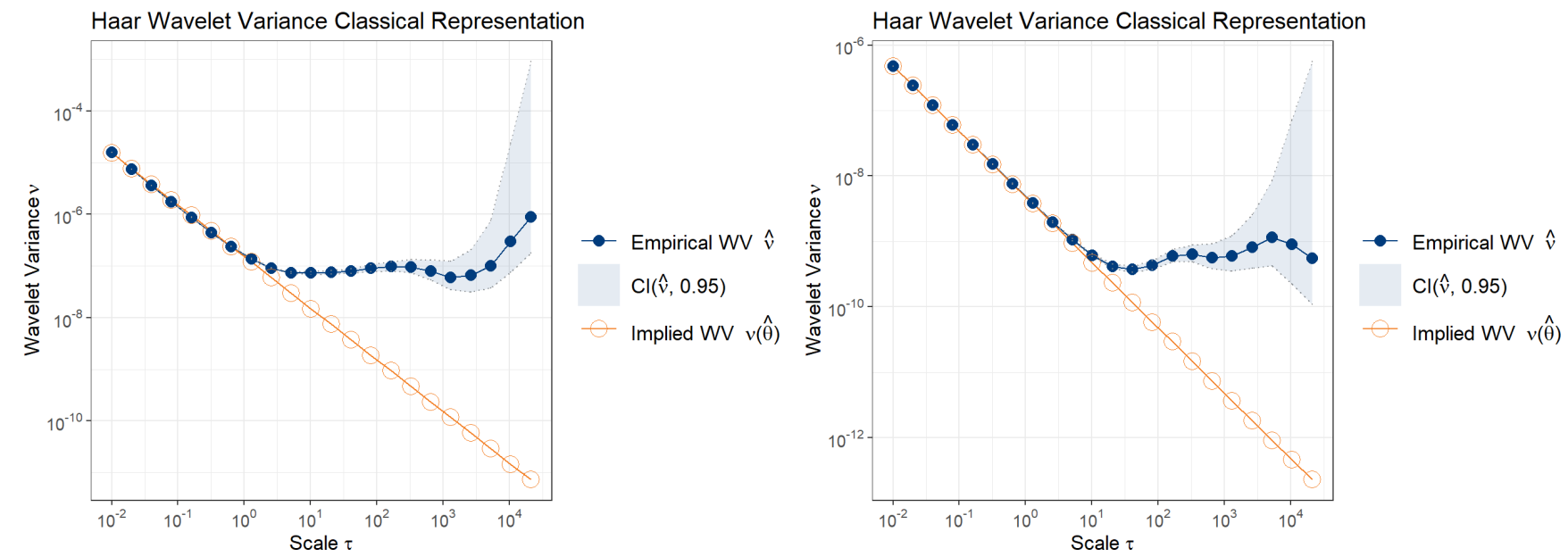

Figure 2: Wavelet Variance plots for sub-optimal Model 1 (orange line w. empty circles) for accelerometers (left) and gyroscopes (right) 

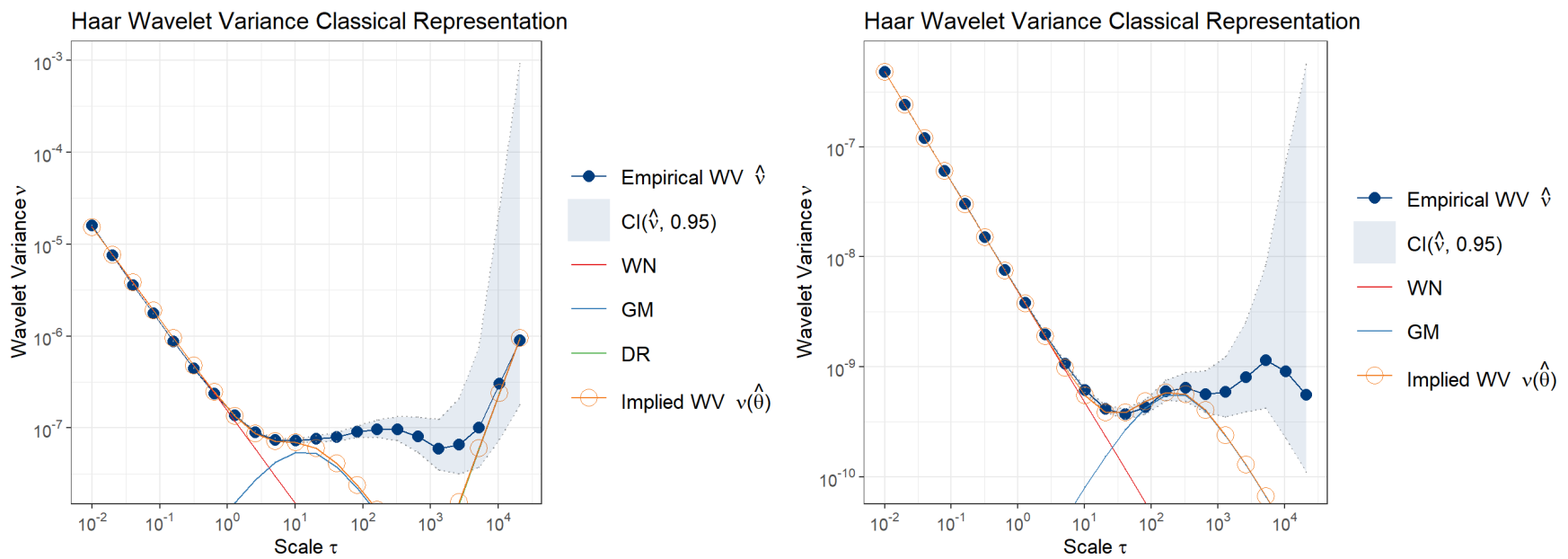

Figure 3: Wavelet Variance plots for Model 2 (orange line w. empty circles) for accelerometers (left) and gyroscopes (right)
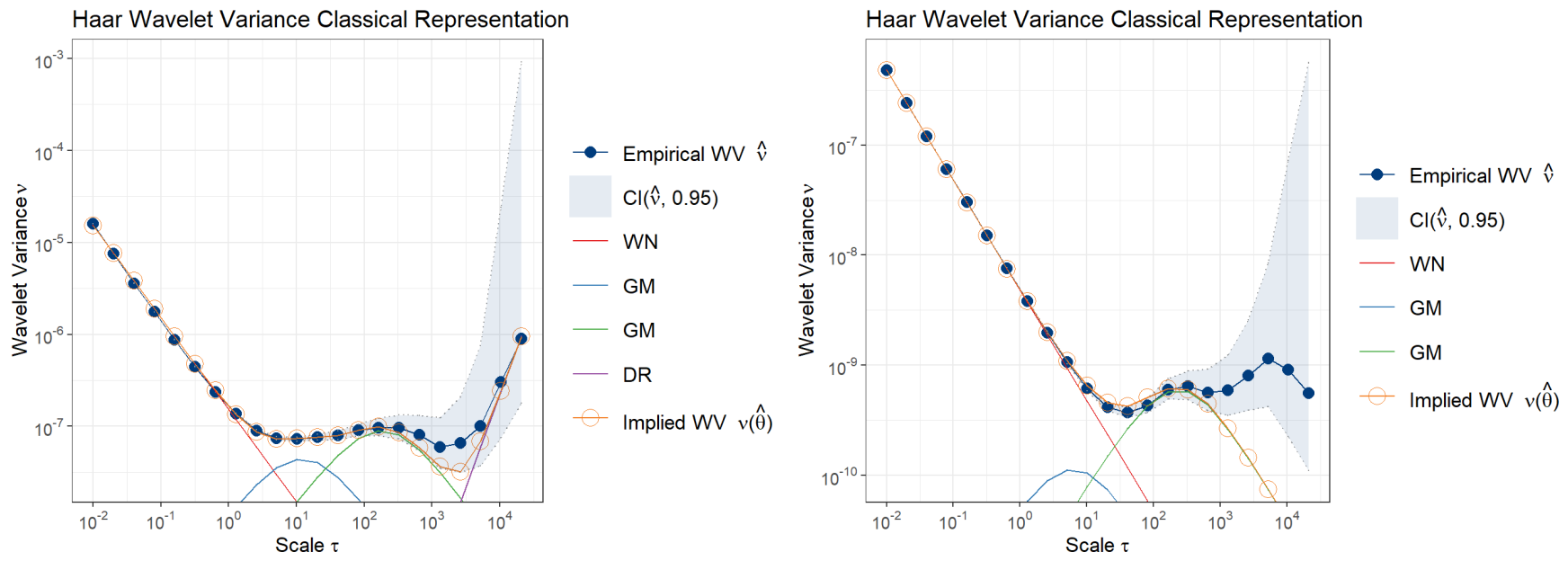

Figure 4: Wavelet Variance plots for Model 3 (orange line w. empty circles) for accelerometers (left) and gyroscopes (right)

Table 1: Parameters of IMU error model (sampling frequency $=200 \mathrm{~Hz}$ )

\begin{tabular}{|c|c|c|c|c|c|c|c|c|c|}
\hline \multirow{2}{*}{\multicolumn{2}{|c|}{ Parameter }} & $\mathrm{WN}$ & \multicolumn{2}{|l|}{ GM 1} & \multicolumn{2}{|l|}{ GM 2} & \multicolumn{2}{|l|}{ GM 3} & \multirow{2}{*}{$\begin{array}{c}\text { DR } \\
\omega\end{array}$} \\
\hline & & $\sigma$ & $\sigma$ & $T$ & $\sigma$ & $T$ & $\sigma$ & $T$ & \\
\hline \multicolumn{2}{|l|}{ Unit } & $m / s^{2} /$ sample & $\mathrm{m} / \mathrm{s}^{2} /$ sample & $s$ & $\mathrm{~m} / \mathrm{s}^{2} /$ sample & $s$ & $\begin{array}{l}m / s^{2} \\
/ \text { sample }\end{array}$ & $s$ & $\begin{array}{l}m / s^{2} \\
/ \text { sample }\end{array}$ \\
\hline \multirow{4}{*}{$\begin{array}{l}\text { Accelerometer } \\
\text { models }\end{array}$} & 1 & $5.526 \times 10^{-3}$ & & & & & & & \\
\hline & 2 & $5.521 \times 10^{-3}$ & $5.413 \times 10^{-4}$ & 3.55 & & & & & $\begin{array}{l}9.306 \\
\times 10^{-10}\end{array}$ \\
\hline & 3 & $5.521 \times 10^{-3}$ & $4.814 \times 10^{-4}$ & 3.21 & $6.874 \times 10^{-4}$ & 49.00 & & & $\begin{array}{l}9.319 \\
\times 10^{-10}\end{array}$ \\
\hline & Ref. & $5.521 \times 10^{-3}$ & $4.811 \times 10^{-4}$ & 3.22 & $6.666 \times 10^{-4}$ & 47.40 & $4.003 \times 10^{-4}$ & 556 & $\begin{array}{l}9.302 \\
\times 10^{-10}\end{array}$ \\
\hline \multicolumn{2}{|l|}{ Unit } & $\mathrm{rad} / \mathrm{s} / \mathrm{sample}$ & $\mathrm{rad} / \mathrm{s} / \mathrm{sample}$ & $S$ & $\mathrm{rad} / \mathrm{s} / \mathrm{sample}$ & $S$ & $\begin{array}{l}\mathrm{rad} / \mathrm{s} \\
\text { /sample }\end{array}$ & $S$ & $\begin{array}{l}\text { rad/s } \\
\text { /sample }\end{array}$ \\
\hline \multirow{4}{*}{$\begin{array}{l}\text { Gyroscope } \\
\text { models }\end{array}$} & 1 & $9.791 \times 10^{-4}$ & & & & & & & \\
\hline & 2 & $9.789 \times 10^{-4}$ & $5.492 \times 10^{-5}$ & 59.29 & & & & & \\
\hline & 3 & $9.788 \times 10^{-4}$ & $5.607 \times 10^{-5}$ & 63.61 & $2.445 \times 10^{-5}$ & 1.665 & & & \\
\hline & Ref. & $9.788 \times 10^{-4}$ & $4.805 \times 10^{-5}$ & 59.22 & $2.209 \times 10^{-5}$ & 1.457 & $7.555 \times 10^{-5}$ & 2008 & \\
\hline
\end{tabular}




\section{INS-BASED INTEGRATED NAVIGATION SYSTEM}

To perform a demo analyses on the effects of IMU error models on navigation solution in this paper, we employ an INS-based navigation system utilizing Extended Kalman Filter (EKF) and few trajectories. As discussed in Sections "Covariance Matrix Analysis" and "Emulation Analysis", we make use of GNSS-derived positions and velocities (when available) and barometer/altimeter measurements within the system. As such a navigation system is well established and can be found in many text books [1], [8], [10], we avoid repeating its description here and refer the interested reader to [11] for its precise description.

\section{COVARIANCE MATRIX ANALYSIS}

In this section, we investigate the evolution of navigation solution uncertainty as a function of IMU error model. Since in general this depents on the trajectory, we follow the standard procedure in INS error analysis [1], [8] by performing covariance propagation considering static conditions first, second dynamic situation represented by a typical trajectory of a small fixed-wing UAV performing mapping mission.

In order to isolate the effect of IMU random errors and its modeling, we discard other sources possibly affecting uncertainty estimation, such as errors due to initialization, deterministic errors, cross-axis errors or gravity modelling errors. Furthermore, as in most practical navigation systems altitude is relatively well-constrained by autonomous means, we have considered near-perfect measurements on altitude to reflect this reality. These measurements usually come from barometers in case of aerial applications and from surface models in case of terrestrial and marine applications. It is worth mentioning that the analysis can be of course performed without such constraint by discarding altitude-related observations.

Figure 5 shows the predicted uncertainty of navigation - PVA (Position, Velocity, Attitude) parameters using different error models for accelerometer (Table 1) and considering no errors in (i.e. perfect) gyroscopes. The same plots using different gyroscope models combined with no error model for accelerometers are depicted in Figure 6.
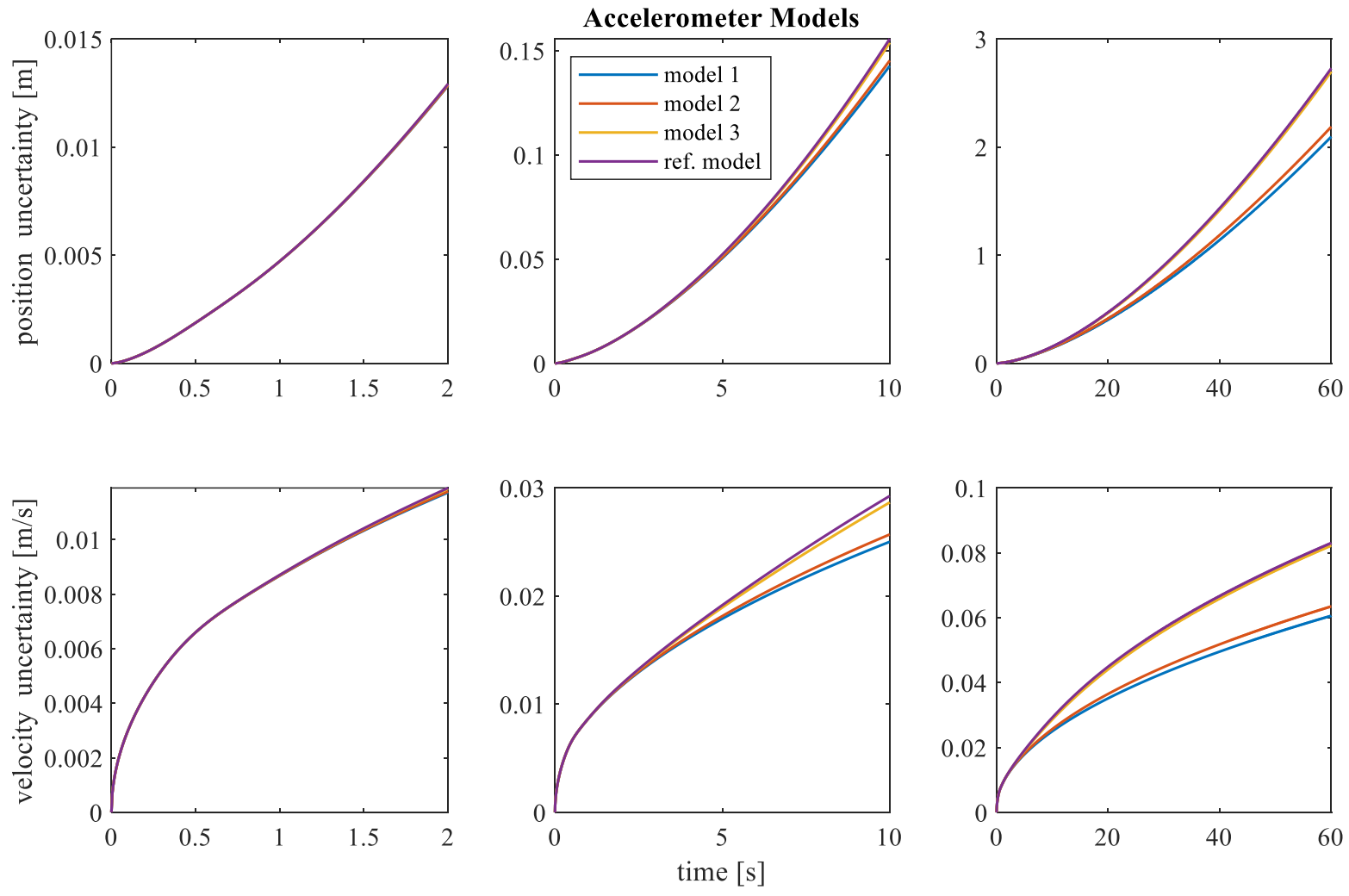

Figure 5: Navigation solution uncertainty growth for different accelerometer error models in static condition 

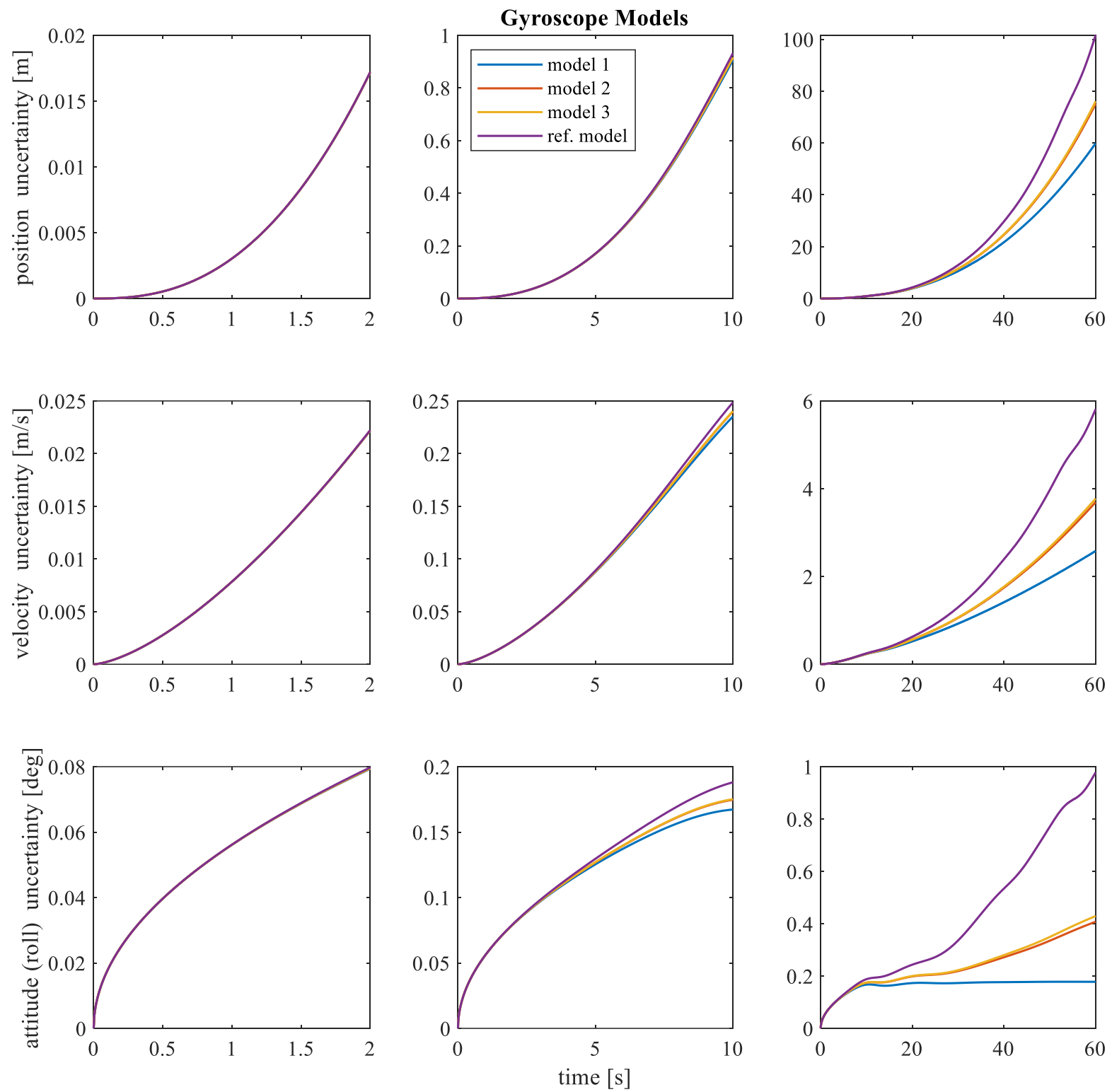

Figure 6: Navigation solution uncertainty growth for different gyroscope error models in static condition

A lot of important observations can be made based on these plots. In general, and as expected, the lower the complexity/fidelity of the error model, the larger the divergence (normally in form of unrealistic optimism) of estimated uncertainty compared to the reference (perfect) model. The covariance propagation based on an error model not representing the reality will on one side lead to an inconsistency with the actual errors, on the other side possibly degrade the overall performance of the navigation system when integrated with other sources of information such as GNSS observations. The latter case will be shown and discussed in next section. In nutshell, an underestimated uncertainty of INS-based solution can lead to suboptimal use of other information sources, through a wrongly reduced Kalman gain for example, and/or increase the risk of rejection of healthy observations.

The degree at which such divergence can be detriment to navigation in practice depends on several factors, among them on the duration and availability of other information sources so that a particular realization of inertial "sensor bias" due to correlated stochastic process become observable by the navigation filter. For this particular IMU, for the interval of short duration (e.g. few seconds) there is no meaningful difference among different models, as in short-term the white noise is the dominant error source which it is comprised within all models. The GM processes have correlation times of generally greater than few seconds (see Figure 1 and Table 1) and after tents of seconds, noticeable differences on accuracy prediction appear among different models, most 
evidently for accelerometers in velocity confidence and for gyroscopes in attitude confidence. For the purpose of visualization and with respect to the employed class of IMU the forward error propagation was performed up to 60 seconds. By this time, the difference among different models is clearly noticeable, and even the degree of uncertainty growth is clearly different in some cases, as in the attitude plot for gyroscope models (Figure 6), for example.

Nevertheless, depending on the application in terms of its duration and also the availability of other information sources/updates and/or the lack of error analysis, the designer of a navigation filter may decide to safely opt for simpler models in inertial sensors. The benefits of such simplifications may be for instance in reduction of the computation costs due to lower number of error states thus filter dimension. If none of these concerns are significant the designer may choose using more realistic models of higher complexity, while having in mind that that stochastic models of longer-correlation may become observable (and then possibly compensated within the navigation system) only under certain time and dynamics.

\section{EMULATION ANALYSIS}

In this section, we opt for a more realistic conditions in terms of application on a specific trajectory. We consider a real trajectory that is typical for aerial imaging using small fixed-wing UAVs as a base for our emulation. Such application is likely to hosts an IMU considered in this paper either for the purpose of navigation/guidance or image orientation. The trajectory is shown in Figure 7.
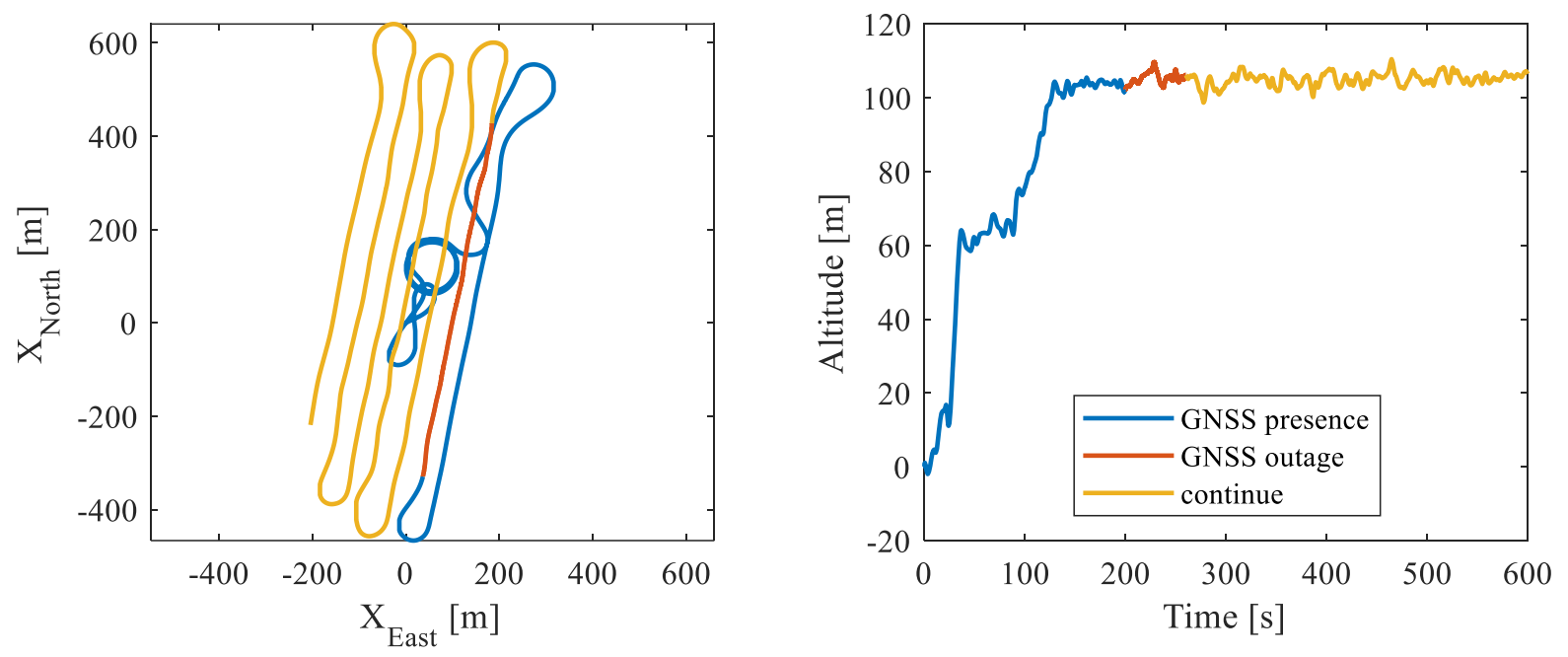

Figure 7: The real trajectory used in emulation analysis

The real trajectory is used to calculate the perfect (error free) inertial measurements from a hypothetical perfect IMU as if it was onboard when executing that trajectory). This trajectory is then used to calculate the perfect (error free) inertial measurements from a hypothetical perfect IMU as if it was onboard when executing that trajectory. The perfect measurements are then contaminated by real random errors of the considered IMU collected in static condition. This singles out the effects or random error modeling from other possible error sources mentioned previously (e.g. errors due to initialization, deterministic errors, cross-axis errors or gravity modelling errors. Within an integrated navigation system then, these realistic measurements are fused with emulated GNSS (at $1 \mathrm{~Hz}$ ) and barometer (at $10 \mathrm{~Hz}$ ) measurements with the error models as presented in Table 2.

Table 2: Error statistics for GNSS and Barometer measurements

\begin{tabular}{|c|c|c|c|c|c|}
\hline \multirow{4}{*}{ White noise $\sigma$} & \multicolumn{2}{|c|}{ GNSS position } & \multicolumn{2}{c|}{ GNSS velocity } & Barometer \\
\cline { 2 - 6 } & $\begin{array}{c}\text { horizontal } \\
\text { (each channel) }\end{array}$ & vertical & $\begin{array}{c}\text { horizontal } \\
\text { (each channel) }\end{array}$ & $0.10 \mathrm{~m} / \mathrm{s}$ & $0.5 \mathrm{~m}$ \\
\cline { 2 - 6 }
\end{tabular}

As long as GNSS measurements are available regularly, the difference IMU error models is hardly noticeable (apart predicting overoptimistic confidence levels). Therefore, we simulate a GNSS outage of 60 seconds duration, during which the drift in navigation solution is compared for different model. 
Figure 8 shows the error grow in navigation solution and its predicted uncertainty by the filter employing different accelerometer models and considering no error model for the gyroscopes. The same plots using different gyroscope models combined with no error model for accelerometers are depicted in Figure 9.
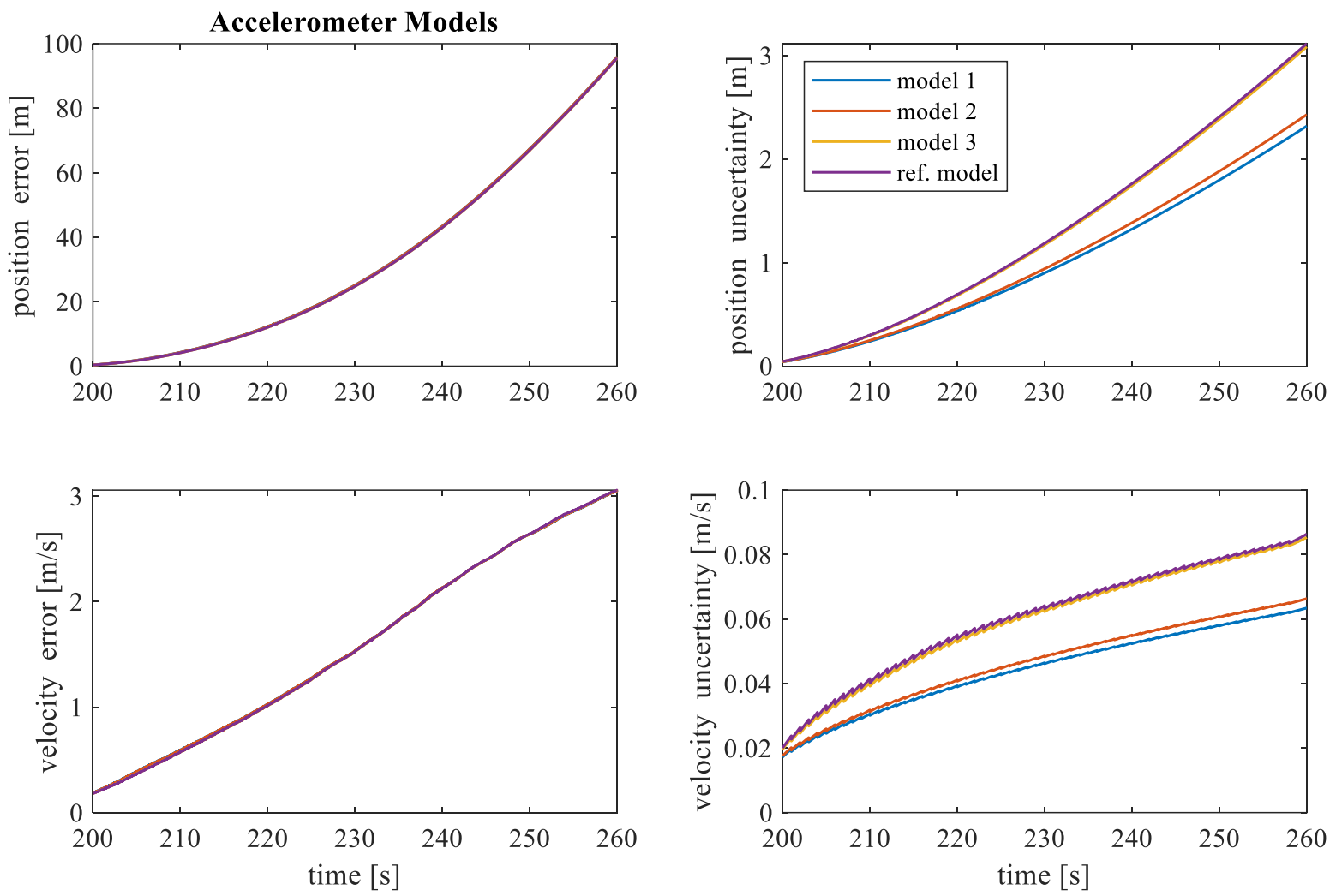

Figure 8: Navigation solution error growth and predicted uncertainty for different accelerometer error models

The first noticeable element in Figure 8 is the large inconsistency between real and predicted error, which is expected since for our IMU the error in gyroscopes has a far more effect on the results, as previously established and discussed in section on "Covariance Matrix Analysis". Therefore, when this error is present in the emulated sensor data, but the associated error model is neglected in the navigation filter, the actual sensor error is projected into navigation error while the filter is unable to predict such projection when considering only accelerometer errors. For the same reason related to the dominance of gyroscope errors, different accelerometer error models hardly affect the actual error in navigation, although there is a clear distinction among them in terms of predicted uncertainty. We can also notice that the models 1 and 2 lead to very similar results while being distinct from those of model 3 and the reference model that are again similar. This means that absence of the GM process (with around $50 \mathrm{~s}$ correlation time) in models 1 and 2 as compared to model 3 and reference model plays a role that is more important with respect to other stochastic processes. Inspecting to the navigation parameters and its confidence levels (Figure 9) for different gyroscope models, we can constant good consistency between actual and predicted navigation errors, fact of which is again associated with the dominance of gyroscope errors for this particular IMU. Also, as expected, the usage of more complex models results in lower actual errors and more realistic (higher in this case) prediction of the PVA confidence level. The only exception to this is the actual error in attitude where it is not easy to distinguish between different models. This may be very specific to this trajectory and realization, though.

Finally, it is worth mentioning that the observations from this specific emulation are in general agreement with the ones resulted from the covariance matrix analysis at static conditions. Therefore, the conclusion on choosing the optimal model remains the same; In general, one can go for the more complex models, but whether the benefits of such models are considerable for the intended application needs to be investigated beforehand. 

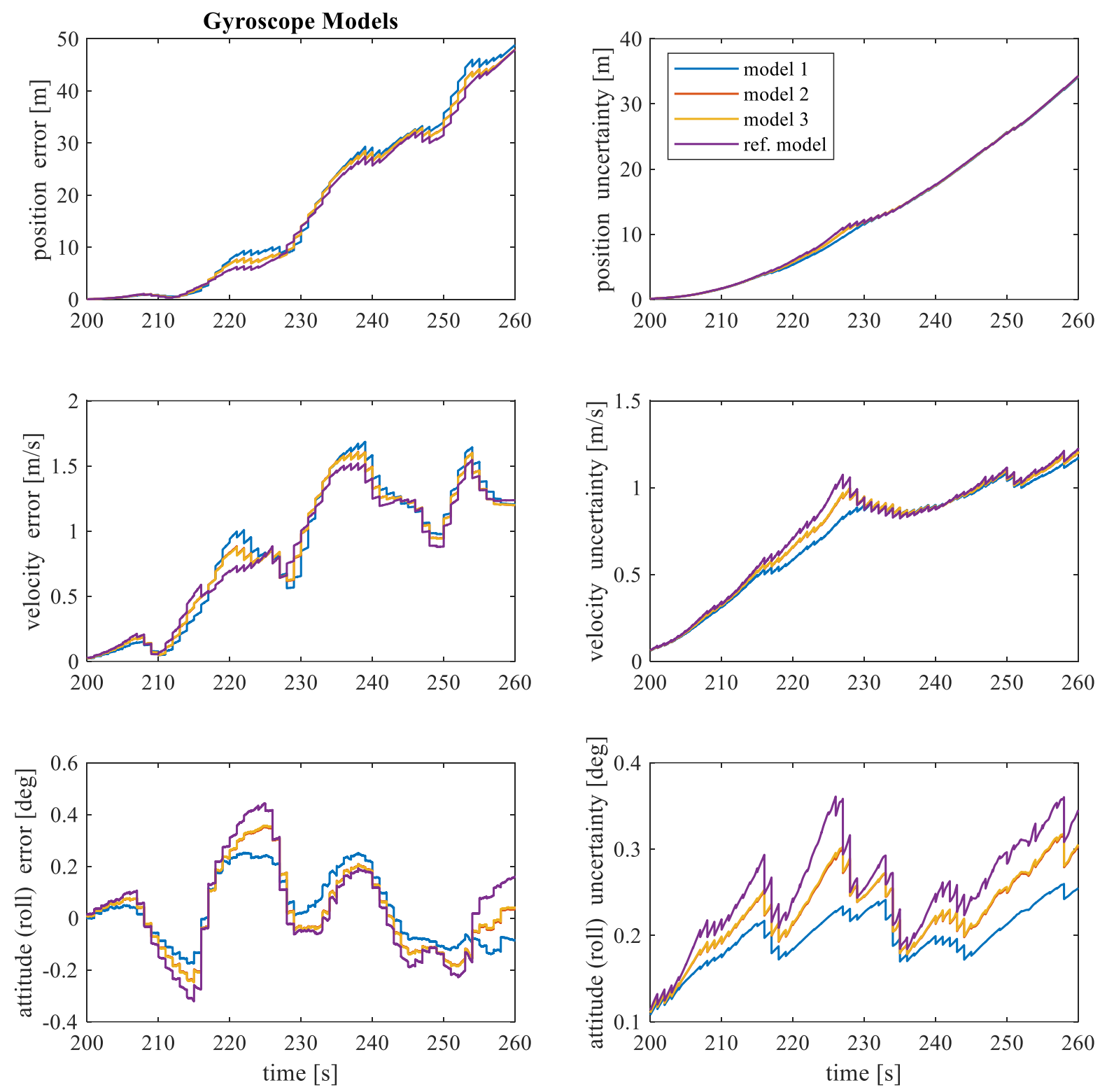

Figure 9: Navigation solution error growth and predicted uncertainty for different gyroscope error models

\section{CONCLUSION}

Stochastic error modeling for inertial sensors remain a crucial part for the design and implementation of integrated navigation systems. While models of higher complexity / fidelity can generally achieve a better match to statistics of sensor error signals such as Allan variance or wavelet variance, simpler models may be also as effective in terms of impact on the integrated navigation performance with less computation cost and easier implementation. More complex models also require more time and higher dynamics for observability.

In this contribution, we studied the impact of IMU error models of different complexity / fidelity as obtained by GMWM analysis on actual navigation performance. We used a high-quality MEMS IMU (Navchip V2), and considered 4 models of different complexity for accelerometers and 4 for gyroscopes. As a first and more general analysis, we performed a covariance matrix analysis in static conditions, which is the standard scenario for error analysis of inertial navigation systems. Results revealed that with more complex models at least 10 seconds is needed to observe a meaningful difference compared to simpler models. Depending on an application, one may never reach such periods of standalone inertial navigation before updates from other sources such as GNSS 
become available. In such cases, therefore, simple models may be sufficient and bring advantages in terms of navigation filter design and implementation. Also, for this particular IMU, gyroscope errors modeling plays a far more important role than that of accelerometers in terms of impact on navigation solution.

As the next step, we performed an emulation study. We used a real trajectory, typical in aerial mapping, flown by a small fixed-wing UAV to obtain sensor data of hypothetical error free sensors as if they were onboard when flying that trajectory. We then used real random sensor error signals to contaminate those ideal signals to study the effect of random error modeling. We simulated a 60second GNSS outage after 200 seconds of GNSS presence, and observe actual navigation error and predicted covariance matrix by the navigation filter. The results of this analysis were in general agreement with those performed in static conditions. As expected, a model of higher fidelity (and complexity) lead to lower error in navigation solution and a more realistic (larger) predicted covariance. Again, the benefits brought by more complex models were observed to be marginal in short prediction / GNSS-outage periods, while quite considerable in longer (tens of seconds). This confirms again the necessity of specifying the application conditions when deciding on the complexity level of inertial sensor error models.

\section{REFERENCES}

[1] P. D. Groves, Principles of GNSS, inertial, and multisensor integrated navigation systems. Artech house, 2013.

[2] S. Guerrier, J. Skaloud, Y. Stebler, and M.-P. Victoria-Feser, "Wavelet-variance-based estimation for composite stochastic processes," J. Am. Stat. Assoc., vol. 108, no. 503, pp. 1021-1030, 2013.

[3] Y. Stebler, S. Guerrier, J. Skaloud, and M.-P. Victoria-Feser, "Generalized method of wavelet moments for inertial navigation filter design,” IEEE Trans. Aerosp. Electron. Syst., vol. 50, no. 3, pp. 2269-2283, Jul. 2014.

[4] A. Radi, G. Bakalli, N. El-Sheimy, S. Guerrier, and R. Molinari, "An Automatic Calibration Approach for the Stochastic Parameters of Inertial Sensors," presented at the 30th International Technical Meeting of The Satellite Division of the Institute of Navigation (ION GNSS+ 2017), Portland, Oregon, 2017, pp. 3053-3060.

[5] SMAC-Group, "GMWM R package." [Online]. Available: https://github.com/SMAC-Group/gmwm.

[6] P. Clausen, J. Skaloud, R. Molinari, J. Balamuta, and S. Guerrier, "An overview of a new sensor calibration platform," in 2017 IEEE International Workshop on Metrology for AeroSpace (MetroAeroSpace), Padua, Italy, 2017, pp. 364-368.

[7] "IEEE Standard Specification Format Guide and Test Procedure for Single-Axis Interferometric Fiber Optic Gyros," IEEE Std 952-1997, pp. 1-84, Feb. 1998.

[8] D. H. Titterton and J. L. Weston, Strapdown inertial navigation technology. Stevenage, U.K., 1997.

[9] Intersense, "Navchip V2 Datasheet," 2018. [Online]. Available: http://www.intersense.com/pages/16/246/.

[10] A. Gelb, Applied Optimal Estimation. Cambridge, MA: The M.I.T. Press, 1988.

[11] M. Khaghani, "Vehicle Dynamic Model Based Navigation for Small UAVs," PhD Thesis, EPFL, Lausanne, Switzerland, 2018. 